\title{
Accuracy improvement of the inSAR quality-guided phase unwrapping based on a modified PDV map.
}

\author{
Tarek Bentahar ${ }^{*}$, Atef Bentahar ${ }^{+}$, Riad Saidi $^{*}$ and Hichem Mayache ${ }^{*}$ \\ Larbi Tebessi University, * LABGET, ${ }^{+}$LAMIS, Constantine Street 12002, Tebessa,Algeria
}

Received $31^{\text {st }}$ of March 2020; accepted $22^{\text {nd }}$ of July 2021

\begin{abstract}
In this paper, an accuracy improvement of the quality-guided phase unwrapping algorithm is proposed. Our proposal is based on a modified phase derivative variance which provides more details on local variations especially for vital patterns such as fringes and edges, hence distorted regions may be re-unwrapped according to this new reliable PDV. The proposed improvement is not only effective on accuracy but also on time, the obtained results have shown that the running time with our proposal is less than that of a skillful optimizationbased algorithm. In order to validate the effectiveness of the proposed approach, the experimental test is carried out on simulated and real data, then a comparison is conducted using several relevant criteria.
\end{abstract}

Key Words: inSAR inteferogram, quality-guided, branch-cut, optimization-based phase unwrapping.

\section{Introduction}

For several decades, researchers have taken great interest in monitoring and acquiring information on the Earth's surface, it is considered as one of the important radar applications [1]. Compared to the optical imaging system, radar imaging is an active system with its own illumination source (Electromagnetic wave). This propriety makes the system able to work day, night, and in all weather conditions. Among radar imaging systems we find interferometric synthetic aperture radar (inSAR), this type has the ability to exploit the phase information using the interferometry technique. Its operating principle is based on double acquisition of the backscattered signal using either two antennas separated by a baseline in a single pass (called one-pass mode), or an antenna and two passes (called multi-pass mode) [2]-[4]. In multi-pass mode and based on the temporal de-correlation generated, this mode is suitable for applications of surface change or deformation detection [5] [6]. Whereas, the spatial de-correlation is exploited in one-pass mode which makes it suitable for earth mapping and digital elevation model (DEM) generation [7]-[9].

Generating the DEMs is impossible without an initial step called the phase unwrapping (PU). It is a key process used to calculate the true phases which are proportional to the real altitudes from the measured phases [10]-[12]. All phases measured by the inSAR system are wrapped in $[-\pi, \pi]$ interval which gives the fringe pattern to the generated interferograms. Mainly, PU is categorized in three classes: single baseline, multi baseline, and large scale PU [11]. Focusing on the single baseline PU, this category can also be divided in three main sub-categories: path following methods; which includes two other classes called branch-cut

Correspondence to: <tarek.bentahar@univ-tebessa.dz>

Recommended for acceptance by $<$ Angel D. Sappa $>$

https://doi.org/10.5565/rev/elcvia. 1220

ELCVIA ISSN: 1577-5097

Published by Computer Vision Center / Universitat Autonoma de Barcelona, Barcelona, Spain 
and quality-guided, optimization-based methods, and integrated de-noising \& unwrapping methods. Each category presents some advantages and disadvantages, but the common perspective is: the more efficient the method in terms of accuracy, the greater the running time.

For the quality-guided techniques, the quality map is an important helper data used to indicate the quality of each pixel measurement. The coherence map (also called correlation coefficient map) is the original quality measurement provided systematically by inSAR system. Other artificial (simulated) maps are numerous and their reliabilities are also different. In [10], it has been shown that the phase derivative variance (PDV) is more efficient than the maximum phase gradient (MPG) especially for steep terrain [13]. Indeed, calculating the quality of a central pixel in a window is more meaningful with the mean of the squares of the deviations from the mean of this window. In other words, all phase differences are involved to calculate the quality. This is not the case for the MPG where only the maximum difference is considered. For this reason we will adopt the PDV map with two modifications in our proposal.

In our proposal, we aim to improve the accuracy of the quality-guided PU to make it close to that of optimization-based methods without increasing the computation time. Therefore, our proposal may offer a compromise between time and accuracy. The paper is organized as following: in section 2 we start with an overview of inSAR system and its generated images principle. In Section 3, the interferogram residues entity and its error propagation are explained. Section 4 is devoted to a short review on the most known PU algorithms. In section 5, improving accuracy based on a modification of the PDV map is presented. Section 6 aims to discuss the obtained results according to widely used criteria. Finally, section 7 concludes the paper and reveals the main deduced points.

\section{Overview of inSAR images}

The working principle of the inSAR imagery system is based on double acquisition of the scattered signal. Using two antennas separated by a baseline, these scattered signals is correlated to eliminate the common phases and to keep only the path phase which is directly related to the imaged point elevation [2][4]. This correlation is called of the system because no de-correlation coefficients are considered, it is noted: $\rho_{\text {system }}=$ Cor $\left[S_{1}, S_{2}{ }^{*}\right]$, where $S_{1}$ and $S_{2}{ }^{*}$ are respectively the master antenna signal and the conjugate of the slave one. To estimate the true correlation coefficient, the spatial and the temporal de-correlation coefficients must be taken in consideration. The spatial de-correlation can be calculated [14] using equation (1), and the temporal one is estimated [15] using equation (2). The true correlation is then: $\rho=\rho_{\text {system }} \rho_{\text {spatial }} \rho_{\text {temporal }}$.

$$
\begin{gathered}
\rho_{\text {spatial }}=1-\frac{2 \operatorname{Rr}\left(\theta_{1}-\theta_{2}\right) \cos \left(\theta_{2}\right)}{\lambda} \\
\rho_{\text {temporal }}=\exp \left[-\frac{1}{2}\left(\frac{4 \pi}{\lambda}\right)^{2}\left(\left(\sigma_{\mathrm{h}} \sin \left(\theta_{1}\right)\right)^{2}+\left(\sigma_{\mathrm{v}} \cos \left(\theta_{1}\right)\right)^{2}\right)\right]
\end{gathered}
$$

Where $\mathrm{Rr}$ is the range resolution, $\lambda$ is the wave length used, $\theta_{1}$ and $\theta_{2}$ are respectively the incidence angle of the master and the slave antenna, $\sigma_{\mathrm{h}}$ and $\sigma_{\mathrm{v}}$ are respectively the horizontal and the vertical standard deviation of a Gaussian function.

The modulus of $\rho$ is the known magnitude image, in which the pixel intensity presents the backscattered coefficient of the imaged point. To generate the interferometric phase image (also called interferogram) and the coherence map, another complex coefficient $\gamma$ has to be calculated, $\gamma$ is defined as the correlation between the two signals divided by the auto-correlation of each one as it is shown in equation (3).

$$
\gamma=\frac{\operatorname{Cor}\left[\mathrm{S}_{1}, \mathrm{~S}_{2}^{*}\right]}{\sqrt{\operatorname{Cor}\left[\left|\mathrm{S}_{1}\right|^{2}\right] \operatorname{Cor}\left[\left|\mathrm{S}_{2}\right|^{2}\right]}}
$$

In brief, three images can be generated by the inSAR imagery system:

- Magnitude image: modulus of $\rho$. 
- Interferogram (interferometric phase image): argument of $\gamma$.

- Coherence map: modulus of $\gamma$.

Figure 1 shows an example of a realist ERS-1 inSAR images. More details are indicated in table 2 below.

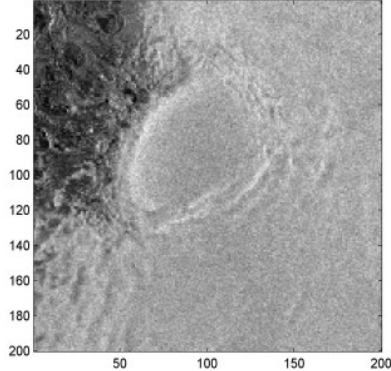

(a)

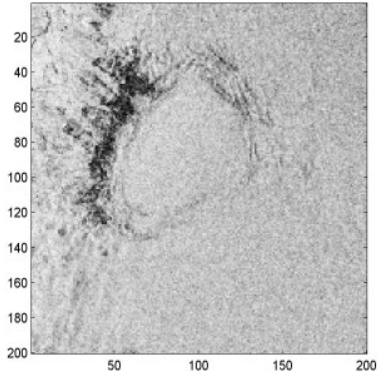

(b)

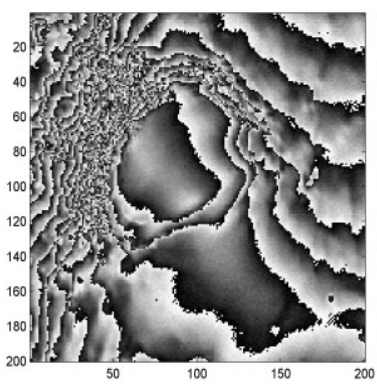

(c)

Fig.1. example of ERS-1 images: (a) magnitude image, (b) coherence map, (c) interferogram

The coherence map is the original inSAR quality measurement, the quality of each pixel can be evaluated in $[0,1]$ range, where 0 is for the worst and 1 is for the best. Using a threshold, the quality of measurement is be segmented in two areas: good and bad overall quality. This segmented coherence is a useful data for some phase unwrapping techniques. Not only for the phase unwrapping, the coherence map can also be used for the de-noising process especially in the frequency field [16]-[18].

In the interferogram, all phases are wrapped into $[-\pi,+\pi]$ interval, which explains the fringe patterns. According to the coherence map, the fringes are homogenous and have an almost stable local frequency in the high coherent regions, contrary in the low coherent regions where these fringes become undistinguished due to the large standard deviation of the measured phase (noise) [2]. The wrapping function; denoted Wrap (.), is defined in equation (4).

$$
\phi_{i, j}^{w}=\operatorname{Wrap}\left(\varphi_{i, j}\right)=\operatorname{Mod}\left\{\left[\pi+\varphi_{i, j}\right], \quad 2 \pi\right\}-\pi
$$

$\varphi_{i, j}$ is the measured interferometric phase of the pixel $(i, j)$ and $\phi_{i, j}^{w}$ is the wrapped one. Mod is the ordinary modulo function.

\section{Residues and error propagation}

Generally, magnitude images are noisy by speckles and interfergorams by residues [2]. The residue is the phase aliasing caused by discontinuities that overstep the half cycle $\pi$, this leads to an important sampling condition called Itoh assumption [19]. The under-sampling is not the only source of residues; they can also be caused by shadow regions, inversion regions or other acquisition system error. The interferogram residues are characterized by their no-uniform distribution which strongly depends on the coherence evaluation (refer to figure 1 and 2). Although the relationship between the coherence and the residues distribution is consistent, the coherence map does not indicate exactly the residues locality. To locate the residue, the wrapped gradients sum of the wrapped phases in $2 \times 2$ loop should to be calculated (refer to equation (5)).

$$
\begin{gathered}
\mathrm{R}=\frac{1}{2 \pi} \sum\left(\operatorname{Wrap}\left(\phi_{i+1, j}^{w}-\phi_{i, j}^{w}\right)+\operatorname{Wrap}\left(\phi_{i+1, j+1}^{w}-\phi_{i+1, j}^{w}\right)+\operatorname{Wrap}\left(\phi_{i, j+1}^{w}-\phi_{i+1, j+1}^{w}\right)\right. \\
\left.+\operatorname{Wrap}\left(\phi_{i, j}^{w}-\phi_{i, j+1}^{w}\right)\right)
\end{gathered}
$$


Since the effective phase discontinuity is a multiple of the cycle number (also called ambiguity number), three values of $\mathrm{R}$ are possible: $\mathrm{R}=\{0,+1,-1\}$. If $\mathrm{R}=0$, the $2 \times 2$ loop is a residue-free area. Else, it is considered as residue. However, the real residue-pixel is not located among the four pixels and only the topleft pixel will be tagged to avoid an overlapped presentation in the residues-map. Figure 2 shows the residues-map of the same interferogarm of figure 1(c). According to $\mathrm{R}$ values, it is to note that this map is a three-value image and not binary. The red and blue pixels correspond to $\mathrm{R}=+1$ and -1 , respectively.

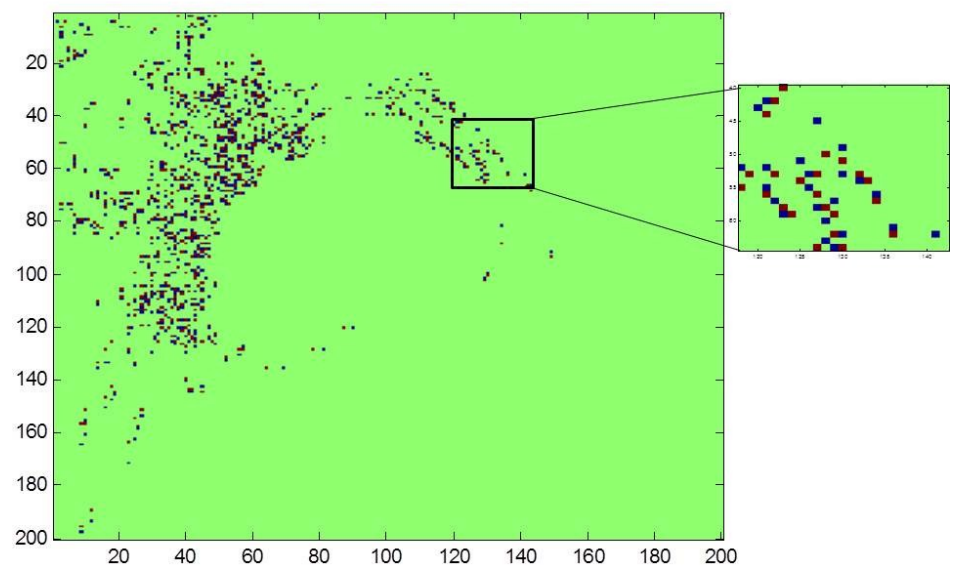

Fig.2. Residues-map of the interferogram of figure 1

The wrapped and unwrapped phases $\phi_{i, j}^{\mathrm{w}}\left(\right.$ and $\phi_{\mathrm{i}, \mathrm{j}}^{\mathrm{u}}$ ) of the pixel $(\mathrm{i}, \mathrm{j})$ are related by a multiple of the cycle number as in Equation (6) where $n(i, j)$ is an index of an unwrapped neighbor of $\phi_{i, j}^{w}$.

$$
\phi_{i, j}^{u}=\phi_{i, j}^{w}+2 \pi \text { Round }\left(\frac{\phi_{n(i, j)}^{u}-\phi_{i, j}^{w}}{2 \pi}\right)
$$

Or, the fact that the gradient of the unwrapped phases is equal to wrapping the gradient of the wrapped phases, the reconstruction can also be achieved using Equation (7).

$$
\phi_{i, j}^{u}=\phi_{n(i, j)}^{u}+\operatorname{Wrap}\left(\phi_{i, j}^{w}-\phi_{n(i, j)}^{w}\right)
$$

In other words, to unwrap a current pixel, any correctly unwrapped neighbor can be used. Otherwise, if the neighbor pixel is mis-unwrapped due to crossing a residue, the error will propagate throughout the interferogram forming distorted lines in the resulted image. The distortion extent depends on the density and the number of these residues. Figure 3 shows an example of the error propagation when the phase unwrapping process crosses the residues area. The used data are simulated interferograms with and without noise of $200 \times 200$ size. 


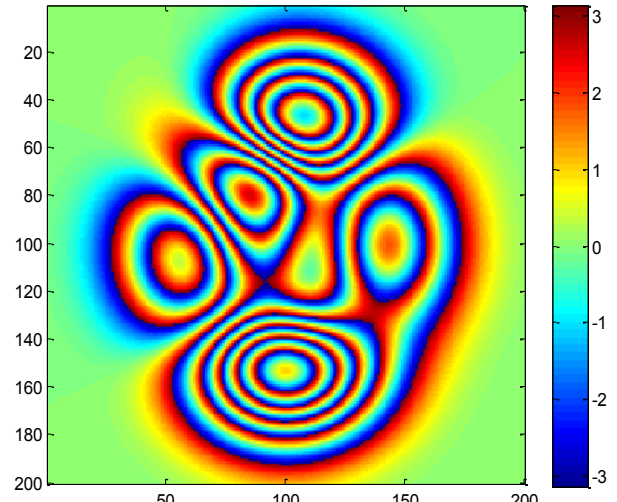

(a)

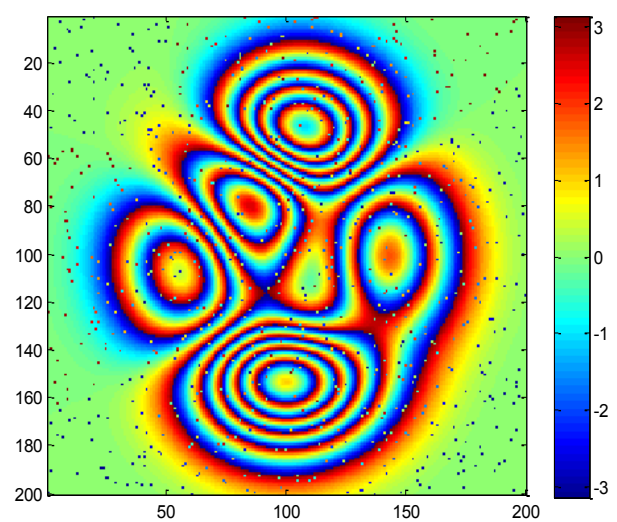

(c)

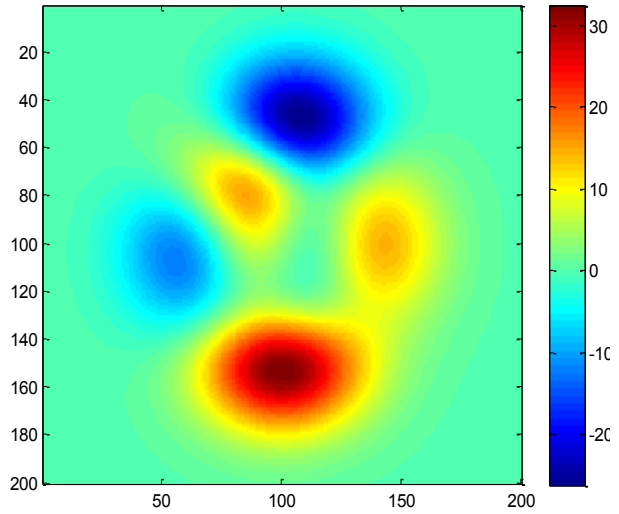

(b)

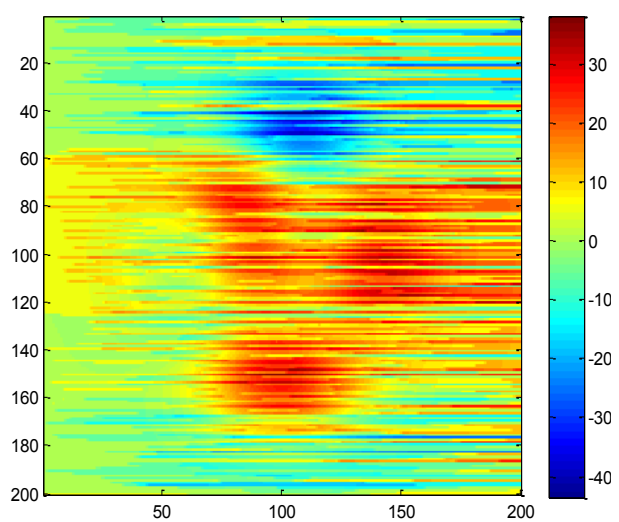

(d)

Fig.3. Error propagation effect, (a) residue-free simulated interferogram, (b) unwrapped image of (a), (c) simulated interferogram with residues, (d) unwrapped image of (c).

\section{Some phase unwrapping techniques}

To avoid the error propagation explained in the previous section, numerous residue-immune phase unwrapping (PU) techniques have been proposed. These methods are divided in three main categories: single-baseline (SBPU), multi-baseline (MBPU), and large-scale (SLPU) [11]. SBPU is also subdivided in three subcategories: path-following (PFPU), optimization-based PU (OPU), and integrated denoising \& unwrapping (IDUPU). All SLPU methods are based on phase continuity and Itoh assumption to locate the unique solution.

In PFPU methods, the integration path should be well chosen in order to find this unique solution and to avoid crossing any noisy area. According to the exploited strategy, PFPU can be classified into two groups: branch-cuts (BC) and quality guided (QG).

$\mathrm{BC}$ methods aim to cover all residues using balanced cuts which link uncharged group of residues, then the flood-fill process will be performed while avoiding these established cuts. The first BC algorithm was proposed by Glodstein [20]. Goldstein's method tries to connect the nearest residues of opposite signs to form the balanced branch-cuts using a growing box. After that, in order to find the minimum total length of branch-cuts, several enhancements and hybridization have been proposed. Among them: branch-cut and finite element PU [21], guided connection of the residues using the pseudo-correlation map [22], Residue- 
Pairing algorithm based on the aggregate of residues coordinates [23], branch-cut based on a simple exchange operation [24], branch-cut based on the spanning-tree algorithm [25], branch-cut and B-Spline Fitting [26], and branch-cut based on a dynamic adjacent table [27].

QG methods aim to unwrap the inteferogram according to its quality; pixels of good quality have priority to be unwrapped before those of bad quality. In another concept, this quality-guided process can be used to mask the noisy region and not to unwrap the good one. In this case, the strategy resembles to that of the branch-cut because it aims to cover the noisy regions. The first QG algorithm was proposed by Roth [28], in which the reliable region to be unwrapped is built by a growing mask based on the quality map. Flynn [29] has inspired from Roth's method his quality-guided PU but in a contrary concept i.e. covering the noisy region by the growing mask. Improved QG algorithms are various, among then we find: [30]-[36].

QG efficiency depends strongly on the quality map exploited [37] [38] which generally evaluates the quality of each pixel in [ $\left.\begin{array}{ll}0 & 1\end{array}\right]$ interval; 0 for the definitively incoherent region and 1 for the highly coherent. The coherence map is the original quality map generated by the inSAR system. If this map is not available, other proposed quality measurement in many literatures can be used such as: phase derivative variance, maximum phase gradient, pseudo correlation, co-occurrence matrix...

As previously mentioned, PDV is better than MPG especially for steep terrain. This advantage comes mainly from the fact that all the phase differences (phase edges) are involved to calculate the quality of the central pixel; this is not the case for MPG. To show this advantage, we have implemented the basic quality guided algorithm described in [10] with the two maps (PDV and MPG). Then, we have calculated for each map the percentage of the discontinuity points in the resulting unwrapped image with the same way described in [13]. The interferogram used for this comparison is the same used in the Results and Discussions section. Table 1 shows the obtained discontinuities which are less with PDV than with MPG.

The comparison between $\mathrm{BC}$ and $\mathrm{QG}$ methods reveals that the isolated and distorted regions (nounwrapped due to high residues density) in BC are larger than in QG. However, BC is faster (especially that of Goldstein) than QG and other categories.

In OPU methods, the finding of the unique solution is not a local process as in the PFPU; it is a global process where all phase values are involved to locate a global optimal solution. The accepted perspective is that a skillful OPU algorithm is accurate compared to PFPU methods but at the expense of the computation time. Costantini's algorithm [39] is one of these methods; in this paper, it will be used as referential algorithm by dint of its accuracy and quality.

\begin{tabular}{|c|c|}
\hline Quality map & $\begin{array}{c}\text { Discontinuity points } \\
\text { percentage }\end{array}$ \\
\hline PDV & $0.62 \%$ \\
\hline MPG & $0.81 \%$ \\
\hline
\end{tabular}

Table 1: Discontinuity points using PDV and MPG

\section{The proposed improvement}

Our proposed accuracy improvement is based on a modified phase derivative variance map. The usual phase derivative variance (PDV) map can be obtained by equation (8).

$$
P D V_{i, j}=\frac{\sqrt{\sum\left(\Delta_{n(i, j)}^{x}-\overline{\Delta_{n(l, j)}^{x}}\right)^{2}}+\sqrt{\sum\left(\Delta_{n(i, j)}^{y}-\overline{\Delta_{n(l, j)}^{y}}\right)^{2}}}{k^{2}}
$$

Where $\mathrm{k}$ is the chosen window size (generally 3 ), $(\mathrm{i}, \mathrm{j})$ is the window center index, $\mathrm{n}(\mathrm{i}, \mathrm{j})$ are the vertical or the horizontal neighbors of $(\mathrm{i}, \mathrm{j})$ in the $\mathrm{k}$ window, $\Delta_{\mathrm{n}(\mathrm{i}, \mathrm{j})}^{\mathrm{x}}$ and $\Delta_{\mathrm{n}(\mathrm{i}, \mathrm{j})}^{\mathrm{y}}$ are respectively the vertical and horizontal partial derivatives of the wrapped phases in the same window, $\overline{\Delta_{\mathrm{n}(1, \mathrm{l})}^{\mathrm{x}}}$ and $\overline{\Delta_{\mathrm{n}(1, \mathrm{l})}^{\mathrm{y}}}$ are respectively the corresponding average of $\Delta_{\mathrm{i}, \mathrm{j}}^{\mathrm{x}}$ and $\Delta_{\mathrm{i}, \mathrm{j}}^{\mathrm{y}}$. This PDV map takes into account the neighbor pixels in the vertical 
and the horizontal directions, the oblique derivatives are not considered in the usual PDV map. In addition, and based on the derivative definition, calculating $\Delta \mathrm{x}$ or $\mathrm{y}$ is always along one direction. To provide more variational details in the local window, the proposed modified PDV takes into consideration all directions including vertical, horizontal, and oblique. Furthermore, the differences (it is preferred to use difference term rather than derivative) are calculated from the center. Figure 4 shows an illustration of the PDV and the modified PDV edge directions. In the modified PDV, 8 phase edges are exploited rather than 4 in the usual PDV. Figure 5 and 6 show respectively the whole PDV maps and their zooms on a fringe line zone of the same real interferogram of figure 1(c). It is noteworthy that the normalized PDV (even the modified one) evaluates the pixel quality in an inverted range of the coherence map i.e. 0 for the best and 1 for the worst.

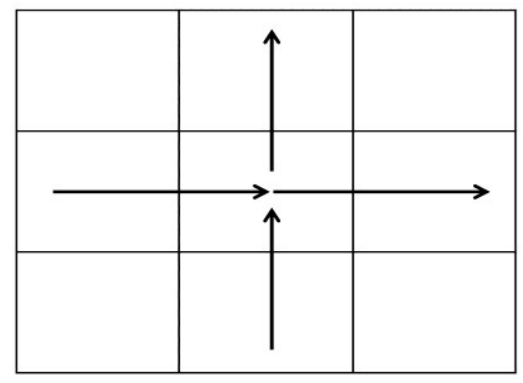

(a)

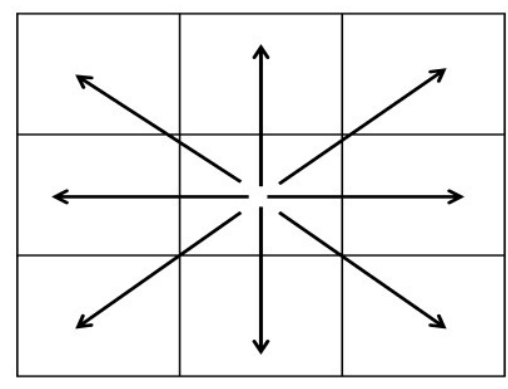

(b)

Fig.4. Phase difference directions in: (a) Usual PDV, (b) Modified PDV.

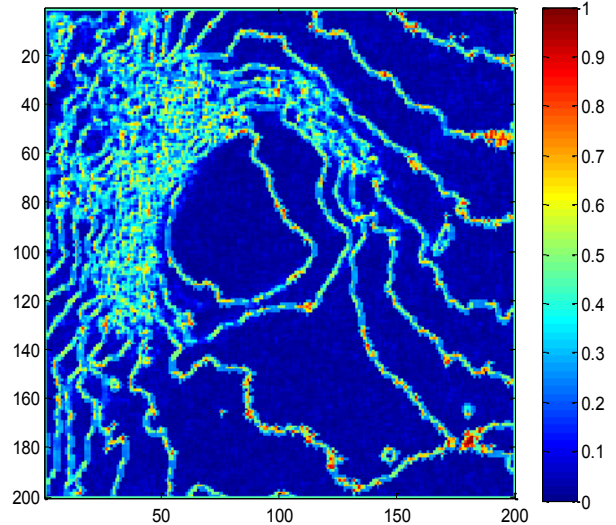

(a)

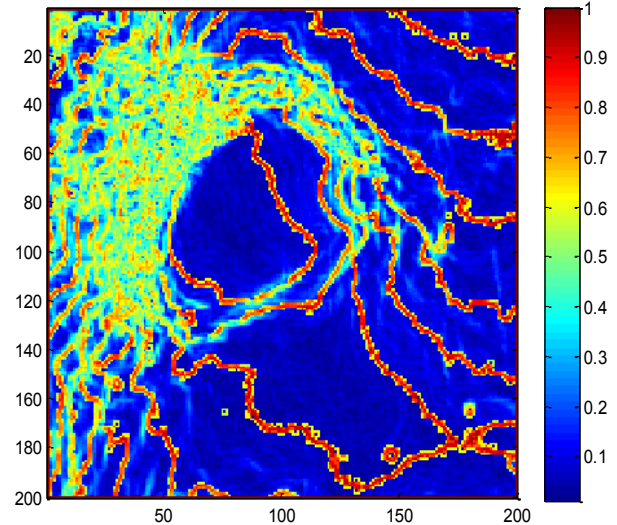

(b)

Fig.5. PDV map of the real interferogram (a) Usual PDV, (b) Modified PDV.

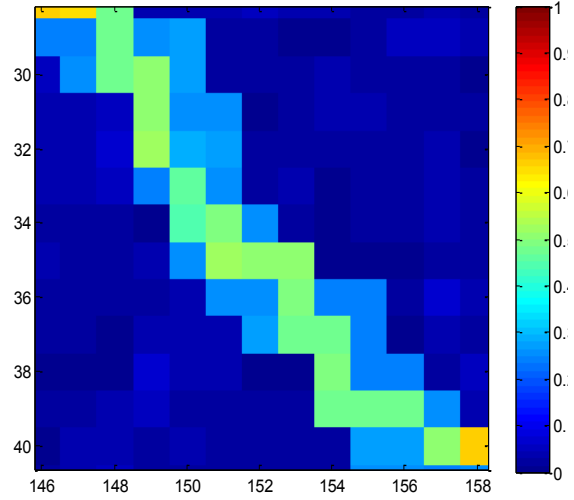

(a)

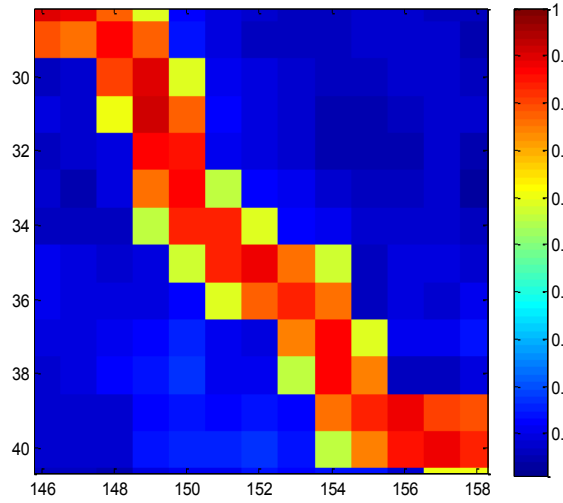

(b)

Fig.6. Zoomed fringe line from : (a) the usual PDV map, (b) the modified PDV map. 
By analyzing figures 5 and 6 , the fringe lines are more highlighted in the modified PDV than in the conventional one. As well as, phase values are also more differentiated which allows providing more details about the local phase values. This propriety can be employed to locate the distorted region and to perform the correctional process in order to enhance the quality of the unwrapped image.

The proposed improvement can be summarized in the following steps:

a- Generate the modified PDV map of the QG phase unwrapped image (not of the interferogram).

b- Assign a threshold to the modified PDV to locate the distorted regions where the quality improvement should be applied.

c- Tag the pixels in the distorted regions to be re-unwrapped.

d- For each tagged pixel

d-1- Re-unwrap the pixel according to the quality of the neighboring pixels in $3 \times 3$ window.

d-2- Discard the pixel from the tagged group.

e- Repeat step "d" until no tagged pixel is found.

For the sub-step (d-1), two strategies are considered. The first one is to re-unwrap the central pixel according to the highest quality neighbor i.e. neighbor with the smallest modified PDV value. This mode is referred to un-weighted unwrapping process because no weights are considered in the calculation of the unwrapped phase. The second strategy is to include all the pixels (in $3 \times 3$ window) already unwrapped with different weights. The weights are chosen with respect to the quality indicated by the modified PDV, the higher the quality, the greater the weight. For simplification purposes, the formulation (9) is used rather than equation (7).

$$
\phi_{i, j}^{u}=\phi_{n(i, j)}^{u}+\operatorname{Wrap}\left(\phi_{i, j}^{w}-\phi_{n(i, j)}^{w}\right)=\operatorname{Unwrap}\left(\phi_{i, j}^{w}\right)_{n(i, j)}
$$

As explained above, this formula means unwrapping the current pixel $(i, j)$ according to the neighbor $n(i, j)$. Therefore, the weighted unwrapping process will be carried out by equation (10).

$$
\phi_{i, j}^{u}=\frac{\sum_{a=1}^{A} W_{a} \cdot \operatorname{Unwrap}\left(\phi_{i, j}^{w}\right)_{n(i, j)_{a}}}{\sum_{a=1}^{A} W_{a}}
$$

Where $W_{a}$ is the weight of the neighbor $n(i, j)_{a}$ and $A$ is the number of all the neighbors already unwrapped in $3 \times 3$ window. If all weights are equal, the process is an average-based un-weighted unwrapping.

\section{$6 \quad$ Results and discussion}

In this implementation section, the real interferogram shown in figure 1(c) and the simulated one of figure 3 (c) are exploited. Their main characteristics and parameters are indicated in table 2 below.

\begin{tabular}{|c|c|c|c|c|c|}
\hline & size & Residues rate & Satellite & Orbit & Imaged region \\
\hline Simulated & $200 \times 200$ & $0.037 \%$ & - & - & - \\
\hline Real & $200 \times 200$ & $0.030 \%$ & ERS-1 & 23315 & Vatnajökull \\
\hline
\end{tabular}

Table 2: Main characteristics and parameters of the test interferograms.

Uniform and no-uniform residues distribution is adopted so that the comparison is credible. Figure 7 shows the residues distribution map of both interferograms. 


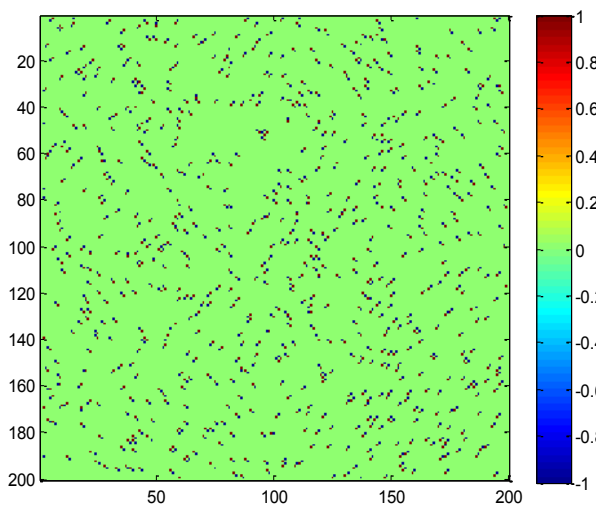

(a)

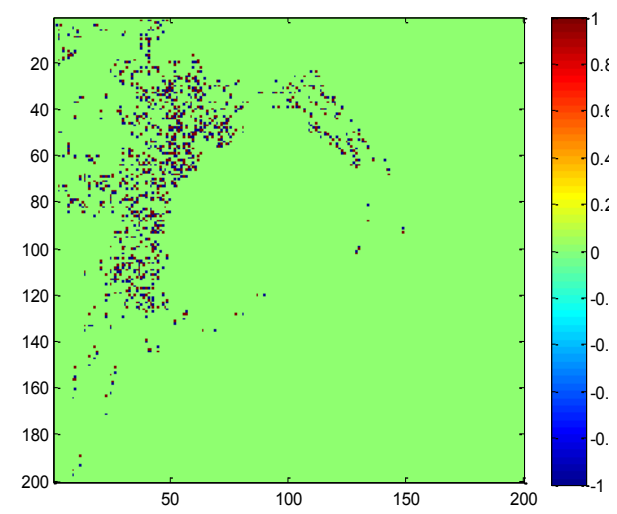

(b)

Fig.7. Residues map of: (a) Simulated interferogram, (b) Real interferogram.

The choice of the phase unwrapping techniques incorporated in our comparison is according to the main categories and to the desired goal of the proposed algorithm. Hence, Goldstein's branch-cut [20], Zhong's quality-guided based on the priority queue [32], Costantini's optimization algorithm [39], Yan's hybrid method [40] and the proposed improvement will be implemented in this section. Goldstein's method is the fastest algorithm at the expense of the accuracy due to the isolated regions (void regions without unwrapping), it serves as a time complexity reference. Zhong's method is the objective of our improvement because it has an acceptable time and accuracy complexity. Costantini's method is the best in terms of accuracy at the expense of the computation time, it serves as an accuracy reference. Yan's method is a recent hybrid technique that combines the ordinary quality-guided and the surface-fitting algorithms to achieve a compromise accuracy-time.

The usual PDV map used in most quality-guided phase unwrapping algorithms is applied on interferogram. In our improvement of this category, the modified PDV map is applied on the resulted unwrapped image as it is previously explained. Figure 8 shows the modified PDV map of the unwrapped image using the quality-guided algorithm for both interferograms (simulated and real).

Assigning threshold to the modified PDV allows locating the poor quality regions where the corresponding pixels will be tagged with "1" as shown in figure 9. The used threshold in our example is 0.3 , this parameter is arbitrary and it is chosen according to the distorted region extent desired to be improved. The smaller the threshold, the larger the tagged region.

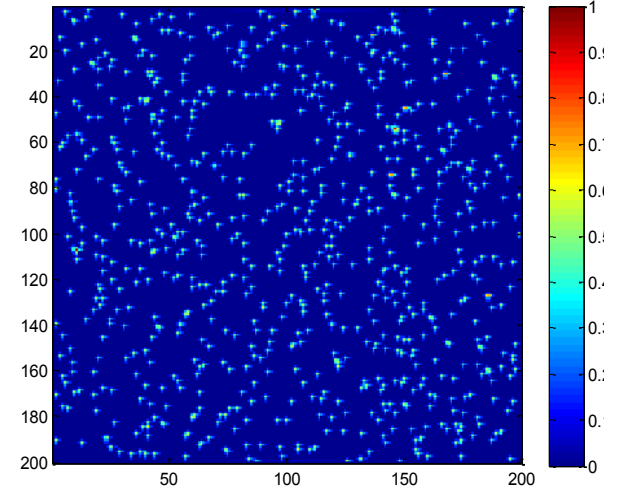

(a)

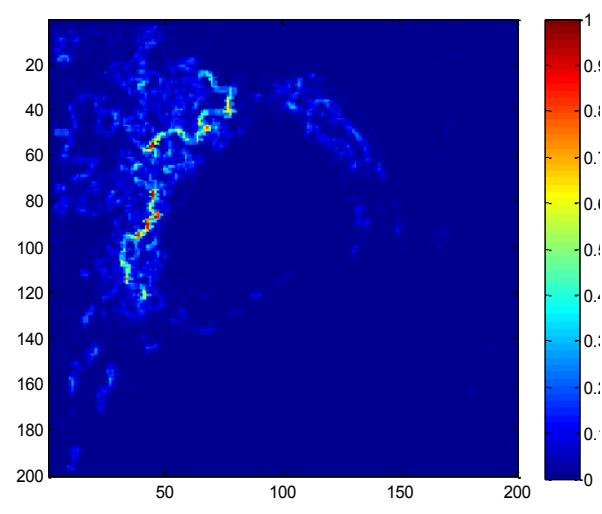

(b)

Fig.8. Modified PDV map of the quality-guided unwrapped image of: (a) the simulated interferogram, (b) the real interferogram. 


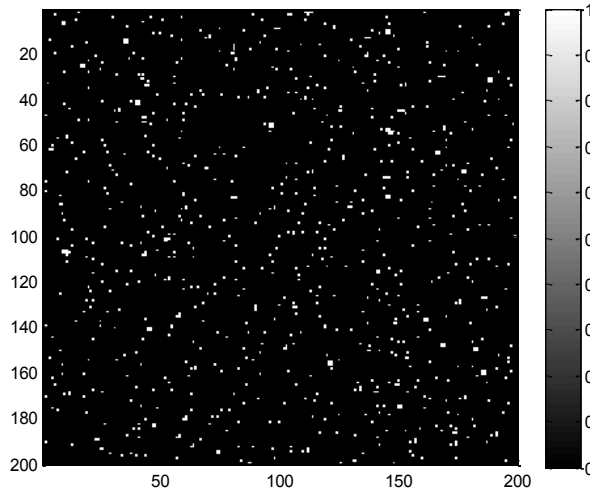

(a)

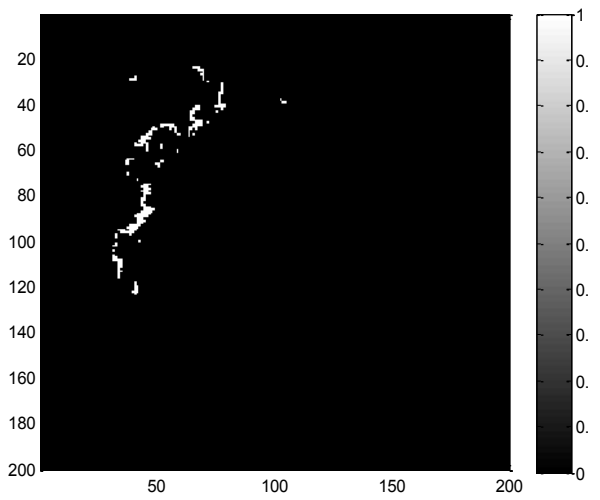

(b)

Fig.9. Tagged pixels to be re-unwrapped with threshold of 0.3: (a) the simulated interferogram, (b) the real interferogram.

Each tagged pixel will be re-unwrapped with respect to the highest quality neighbor in $3 \times 3$ window using simply equation (7) for the un-weighted improvement or using equation (10) for the weighted improvement. Using a PDV map (usual or the modified one), the highest quality pixel is that of the smallest PDV value. After the re-unwrapping, the corresponding pixel is removed (de-tagged) from the list and the process continues to the next one. Trivially, the process should starts from the edges to ensure a sufficient number of the already unwrapped pixels (at least 3 among 8). Figures 10 and 11 show the results of: Glodstein's, Zhong's and Costantini's phase unwrapping methods as well as the proposed accuracy improvement of Zhong's method respectively for the simulated and real interferogram.

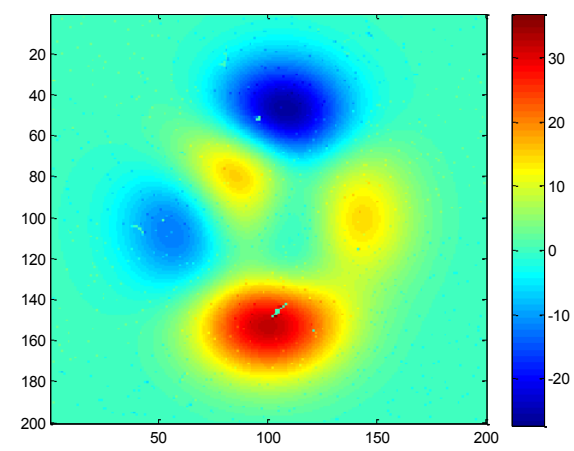

(a)

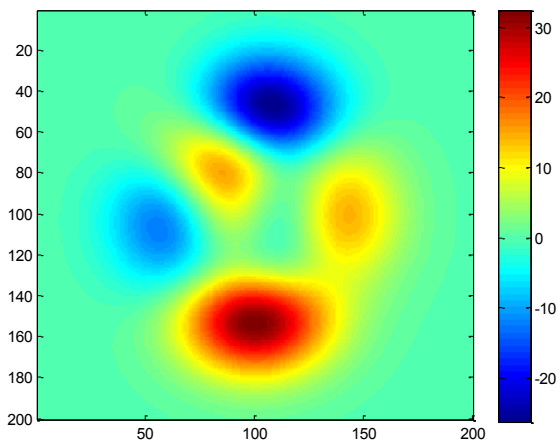

(c)

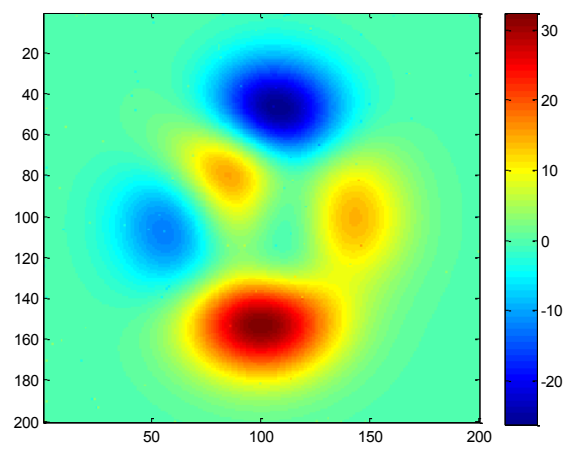

(b)

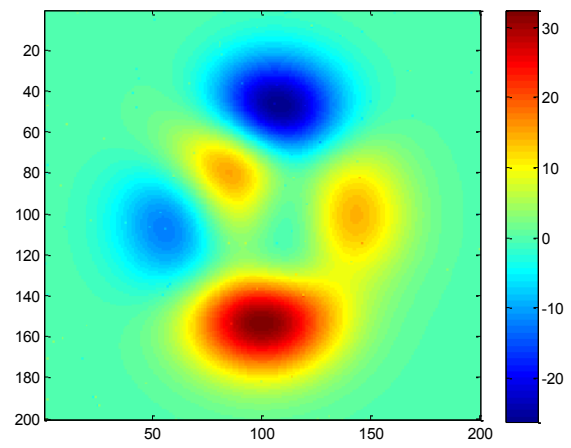

(d)

Fig.10. Unwrapped image of the simulated interferogram using algorithm of: (a) Glodstein, (b)Zhong, (c) Costantini, (d) the proposed improvement. 


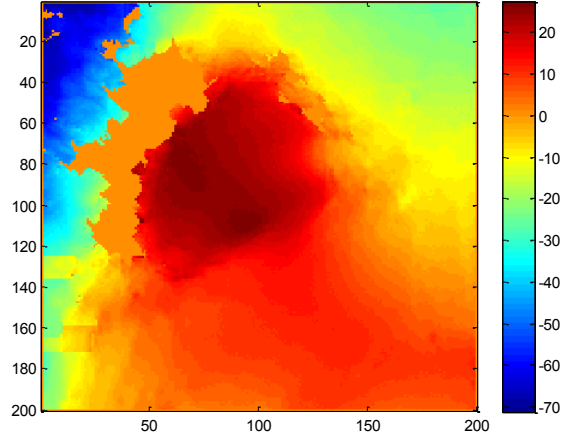

(a)

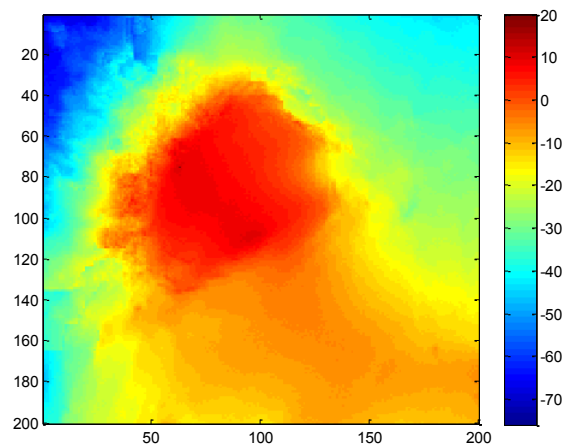

(c)

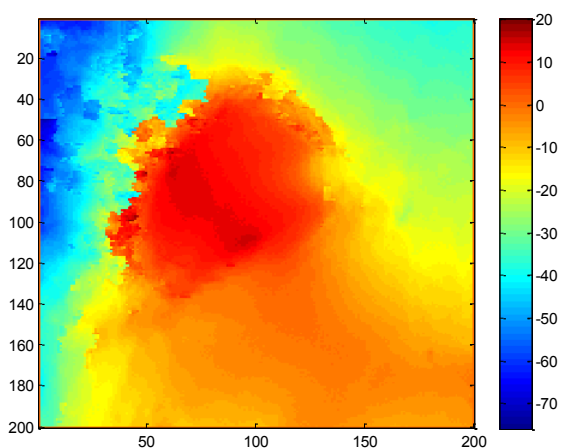

(b)

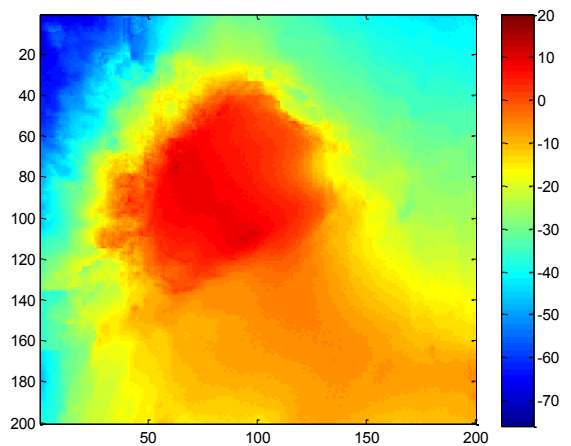

(d)

Fig.11. Unwrapped image of the real interferogram using algorithm of: (a) Glodstein, (b)Zhong, (c) Costantini, (d) the proposed improvement.

Visibly, the image obtained by the proposed improvement has a satisfactory quality and it is quite similar to that of reference (unwrapped by Costantini's algorithm). For a quantitative analysis, numerous metrics can be exploited. In our implementation, Root Mean Squared Error (RMSE), Mean Relative Error (MRE), Standard Deviation $(\sigma)$, Structural Similarity (SSIM) [41], and Discontinuous Point Index (here referred to DPI) [36] are chosen to evaluate the accuracy. As Costantini's method is an accurate algorithm, it can be an accuracy reference and the normalized DPI is preferred to use for a referential comparison.

To show the influence of our proposal in time complexity aspect, the calculated running time of each algorithm is normalized to a time reference which is that of Goldstein's algorithm because it is the fastest. Minimum RMSE, MRE, $\sigma$, DPI, and Time, maximum SSIM are obviously obtained by the best algorithm. However, a good accuracy (good RMSE, MRE, $\sigma$, SSIM, and DPI) is generally occurred at the expense of running time. Table 3 and 4 summarize a comparison of all obtained metrics for the simulated and the real interferograms respectively. According to these results, the proposed improvement changes made the accuracy of quality-guided algorithm (Zhong's method) closer to that of optimization-based algorithm (Costantini's method). Moreover, the proposed improvement is approximately $1.25(6.75 / 5.35$ and 7.56/6.15) and $1.15(6.75 / 6.19$ and 7.56/6.25) faster than the optimization-based algorithm for un-weighted and weighted improvement respectively. Our proposal is also faster than the hybridization algorithm (approximately 1.29 and 1.18 for un-weighted and weighted improvement respectively).

As a comparison between the un-weighted and the weighted improvement, incorporating weights enhances the performance under accuracy criteria but with slight time expense. 


\begin{tabular}{|c|c|c|c|c|c|c|}
\hline & $\begin{array}{c}\text { Glodstein } \\
{[20]}\end{array}$ & $\begin{array}{c}\text { Zhong } \\
{[32]}\end{array}$ & $\begin{array}{c}\text { Costantini } \\
{[39]}\end{array}$ & $\begin{array}{c}\text { Yan } \\
{[40]}\end{array}$ & $\begin{array}{c}\text { Un- } \\
\text { weighted } \\
\text { Improvement }\end{array}$ & $\begin{array}{r}\text { weighted } \\
\text { Improvement }\end{array}$ \\
\hline RMSE (rad) & 0.68 & 0.44 & 0 & 0.21 & 0.18 & 0.19 \\
\hline MRE (\%) & 1.62 & 0.57 & 0 & 0.10 & 0.14 & 0.13 \\
\hline$\sigma(\mathrm{rad})$ & 7.6081 & 7.6038 & 7.5891 & 7.6710 & 7.5897 & 7.5424 \\
\hline SSIM & 0.53 & 0.73 & 1 & 0.91 & 0.89 & 0.89 \\
\hline $\begin{array}{c}\text { Normalized } \\
\text { DPI }\end{array}$ & 1.87 & 1.55 & 1 & 1.47 & 1.17 & 1.14 \\
\hline $\begin{array}{c}\text { Normalized } \\
\text { time }\end{array}$ & 1 & 4.05 & 6.75 & 7.18 & 5.35 & 6.19 \\
\hline
\end{tabular}

Table 3: Obtained results for the simulated interferogram.

\begin{tabular}{|c|c|c|c|c|c|c|}
\hline & $\begin{array}{c}\text { Glodstein } \\
{[20]}\end{array}$ & $\begin{array}{c}\text { Zhong } \\
{[32]}\end{array}$ & $\begin{array}{c}\text { Costantini } \\
{[39]}\end{array}$ & $\begin{array}{c}\text { Yan } \\
{[40]}\end{array}$ & $\begin{array}{c}\text { Un- } \\
\text { weighted } \\
\text { Improvement }\end{array}$ & $\begin{array}{c}\text { weighted } \\
\text { Improvement }\end{array}$ \\
\hline RMSE (rad) & 0.75 & 0.56 & 0 & 0.23 & 0.23 & 0.19 \\
\hline MRE (\%) & 7.09 & 4.04 & 0 & 1.31 & 1.21 & 1.08 \\
\hline$\sigma(\mathrm{rad})$ & 19.013 & 17.784 & 16.147 & 16.153 & 16.146 & 16.153 \\
\hline SSIM & 0.45 & 0.67 & 1 & 0.88 & 0.91 & 0.93 \\
\hline $\begin{array}{c}\text { Normalized } \\
\text { DPI }\end{array}$ & 1.66 & 1.50 & 1 & 1.34 & 1.24 & 1.24 \\
\hline $\begin{array}{c}\text { Normalized } \\
\text { time }\end{array}$ & 1 & 4.58 & 7.56 & 7.64 & 6.15 & 6.32 \\
\hline
\end{tabular}

Table 4: Obtained results for the real interferogram.

\section{Conclusion}

In this paper, an accuracy improvement of the inSAR quality-guided phase unwrapping algorithm is presented. The proposed improvement is based on a modified PDV where important patterns such as fringes and edges are well highlighted compared to the usual PDV. The incorporated modifications are in the directions and the number of phase differences involved. This made it possible to have a more exact estimate of the local variations according to all the neighboring pixels rather than four. This propriety is employed to locate the distorted region, and therefore to perform the correctional process for the quality-guided unwrapped image. This new PDV has proven its ability to offer an accurate re-unwrapping operation for the poor quality phases. Our proposal has not only proven its efficiency on accuracy but also on running time which is less than that of a referential optimization-based algorithm. For a credible comparison, the test is carried out using simulated and real interferograms, and the results are compared using six relevant metrics. The obtained results are satisfactory in term of accuracy and also in term of computation time. 


\section{Acknowledgments}

Authors would like to thank the LABGET and LAMIS laboratories members of Larbi Tebessi University, DGRSTD for the useful SNDL platform. Special thanks to ESA for freely providing ERS inSAR interfergrams.

\section{References}

[1] K. Raney, "Radar, Altimeters," in Encyclopedia of Remote Sensing, Springer New York, pp. 525-532, 2014. https://doi.org/10.1007/978-0-387-36699-9_134.

[2] R. Bamler and P. Hartl, "Synthetic aperture radar interferometry," Inverse Problems, vol. 14, no. 4, pp. R1-R54, Aug. 1998. doi:10.1088/0266-5611/14/4/001.

[3] A. Moreira, P. Prats-Iraola, M. Younis, G. Krieger, I. Hajnsek, and K. P. Papathanassiou, "A tutorial on synthetic aperture radar," IEEE Geoscience and Remote Sensing Magazine, vol. 1, no. 1, pp. 6-43, Mar. 2013. doi: 10.1109/MGRS.2013.2248301.

[4] P. A. Rosen et al., "Synthetic aperture radar interferometry," IEEE Proceedings , vol. 88, no. 3, pp. 333-382, Mar. 2000. doi: 10.1109/5.838084.

[5] B. Osmanoğlu, F. Sunar, S. Wdowinski, and E. Cabral-Cano, "Time series analysis of InSAR data: Methods and trends," ISPRS Journal of Photogrammetry and Remote Sensing, vol. 115, pp. 90-102, May 2016. https://doi.org/10.1016/j.isprsjprs.2015.10.003.

[6] O. Monserrat, M. Crosetto, and G. Luzi, "A review of ground-based SAR interferometry for deformation measurement," ISPRS Journal of Photogrammetry and Remote Sensing, vol. 93, pp. 40-48, Jul. 2014. https://doi.org/10.1016/j.isprsjprs.2014.04.001.

[7] D. Hoja, P. Reinartz, and M. Schroeder," Comparison of DEM Generation and Combination Methods Using High Resolution Optical Stereo Imagery and Interferometric SAR Data”. Revue Française de Photogrammétrie et de Télédétection, 2006 (4), pp. 89-94. 2006.

[8] A. Geymen, "Digital elevation model (DEM) generation using the SAR interferometry technique," Arabian Journal of Geosciences, vol. 7, no. 2, pp. 827-837, Dec. 2012. https://doi.org/10.1007/s12517-012-0811-3.

[9] M. Crosetto, "Calibration and validation of SAR interferometry for DEM generation," ISPRS Journal of Photogrammetry and Remote Sensing, vol. 57, no. 3, pp. 213-227, Dec. 2002. https://doi.org/10.1016/S0924-2716(02)00107-7.

[10] D. C. Ghiglia, ., and D. Pritt. Two-dimensional phase unwrapping: theory, algorithms, and software. Vol. 4. New York: Wiley, 1998.

[11] H. Yu, Y. Lan, Z. Yuan, J. Xu, and H. Lee, "Phase Unwrapping in InSAR: A Review," IEEE Geoscience and Remote Sensing Magazine, vol. 7, no. 1, pp. 40-58, Mar. 2019. doi: 10.1109/MGRS.2018.2873644.

[12] R. Gens, "Two-dimensional phase unwrapping for radar interferometry: Developments and new challenges," International Journal of Remote Sensing, vol. 24, no. 4, pp. 703-710, Jan. 2003. https://doi.org/10.1080/0143116021000016725.

[13] Y. Man, L. Goulin, and H. Huadong, "Comparison among Quality Maps Applying in Phase Unwrapping for Different Terrain," 2010 International Conference on Computational and Information Sciences (ICCIS), Dec. 2010. doi: 10.1109/ICCIS.2010.200.

[14] E. Rodriguez: "Theory and design of interferomtry SAR", IEE Proceedings F - Radar and Signal Processing, Vol. 139 N 2, pp. 147 - 159, 1992. doi: 10.1049/ip-f-2.1992.0018.

[15] H. Zeber: "Decorrelation interferomertic Radar echoes", IEEE transaction on geoscience and remote sensing, Vol. 30 N 5, pp. 950-959, 1992. doi: 10.1109/36.175330.

[16] R. M. Goldstein and C. L. Werner, " Radar interferogram filtering for geophysical applications ", Geophysical Research Lettres, VOL 25, No 21, pp 4035-4038, Nov 1998. https://doi.org/10.1029/1998GL900033. 
[17] I. Baran, M. P. Stewart, B. M. Kampes, Z. Perski, and P. Lilly, "A Modification to the Goldstein Radar Interferogram Filter", IEEE Transactions on Geocience And Remote Sensing, Vol 41, N. 9,pp $2114-$ 2118, Sep 2003. Doi:10.1109/TGRS.2003.817212.

[18] R.Song, H. Guo, G.Liu, Z. Perski, H. Yue, C. Han, and J. Fan, "Improved Goldstein SAR Interferogram Filter Based on Adaptive-Neighborhood Technique", IEEE Transactions on Geocience And Remote Sensing, Vol 12, No 1, pp 140-144, Jan 2015. Doi:10.1109/LGRS.2014.2329498.

[19] K. Itoh, "Analysis of the phase unwrapping problem," Appl. Opt., vol. 21, no. 14, p. 2470, 1982. https://doi.org/10.1364/AO.21.002470.

[20] R. M. Goldstein, H. A. Zebker, and C. L. Werner, "Satellite radar interferometry: Two dimensional phase unwrapping," Radio Science, vol. 23, no. 4, pp. 713-720, Jul. 1988. Doi: 10.1029/RS023i004p00713.

[21] W. Nan and F. Dazheng, "InSAR phase unwrapping algorithm using the brahch-cut and finite element method," in Proceedings 7th International Conference on Signal Processing, 2004. Proceedings. ICSP '04. 2004. Doi: 10.1109/ICOSP.2004.1442151.

[22] X. Feng, W. Jicang, Z. Lei, and L. Xiaoling, "A new method about placement of the branch cut in twodimensional phase unwrapping," in 2007 1st Asian and Pacific Conference on Synthetic Aperture Radar, 2007. Doi: 10.1109/APSAR.2007.4418721

[23] C. Li and D.-Y. Zhu, "A Residue-Pairing Algorithm for Insar Phase Unwrapping," Progress In Electromagnetics Research, vol. 95, pp. 341-354, 2009. doi:10.2528/PIER09070706.

[24] D. Zheng and F. Da, "A novel algorithm for branch cut phase unwrapping," Optics and Lasers in Engineering, vol. 49, no. 5, pp. 609-617, May 2011. https://doi.org/10.1016/j.optlaseng.2011.01.017.

[25] Y. Hanwen, X. Mengdao, and B. Zheng, "A Fast Phase Unwrapping Method for Large-Scale Interferograms," IEEE Transactions on Geoscience and Remote Sensing 51(7), 4240-4248, 2013. Doi: 10.1109/TGRS.2012.2229284.

[26] Z. Yan and X. ZiJian, "A Phase Unwrapping Algorithm Based on Branch-cut and B-Spline Fitting in InSAR," in 2018 IEEE International Conference on Signal Processing, Communications and Computing (ICSPCC), 2018. Doi: 10.1109/ICSPCC.2018.8567735

[27] T. Liu, Z. Shang, J. Wu, D. Zhou, and S. Yan, "Improved branch-cut phase unwrapping strategy based on dynamic adjacent table," The Journal of Engineering, vol. 2019, no. 19, pp. 5805-5809, Oct. 2019. Doi: 10.1049 joe.2019.0352.

[28] Roth, M. W. "Phase unwrapping for interferometric SAR by the least-error path". Johns Hopkins University Applied Physics Lab Technical Report, Laurel, Md, 30, 27. 1995.

[29] T. J. Flynn, "Consistent 2-D phase unwrapping guided by a quality map," IGARSS '96. International Geoscience and Remote Sensing Symposium, Lincoln, NE, USA, vol. 4, pp. 2057-2059, 1996. Doi: 10.1109/IGARSS.1996.516887.

[30] W. Xu and I. Cumming, "A region-growing algorithm for InSAR phase unwrapping," in IEEE Transactions on Geoscience and Remote Sensing, vol. 37, no. 1, pp. 124-134, Jan. 1999. Doi: $10.1109 / 36.739143$.

[31] M. A. Herráez, D. R. Burton, M. J. Lalor, and M. A. Gdeisat, "Fast two-dimensional phase-unwrapping algorithm based on sorting by reliability following a noncontinuous path," Applied Optics, vol. 41, no. 35, p. 7437, Dec. 2002. https://doi.org/10.1364/AO.41.007437.

[32] H. Zhong, J. Tang, S. Zhang, and M. Chen, "An Improved Quality-Guided Phase-Unwrapping Algorithm Based on Priority Queue," IEEE Geoscience and Remote Sensing Letters, vol. 8, no. 2, pp. 364-368, Mar. 2011. Doi: 10.1109/LGRS.2010.2076362.

[33] Z. Dai and X. Zha, "An Accurate Phase Unwrapping Algorithm Based on Reliability Sorting and Residue Mask," IEEE Geoscience and Remote Sensing Letters, vol. 9, no. 2, pp. 219-223, Mar. 2012. Doi: 10.1109/LGRS.2011.2165198.

[34] H. Zhong, J. Tang, S. Zhang, and X. Zhang, "A Quality-Guided and Local Minimum Discontinuity Based Phase Unwrapping Algorithm for InSAR/InSAS Interferograms," IEEE Geoscience and Remote Sensing Letters, vol. 11, no. 1, pp. 215-219, Jan. 2014. Doi: 10.1109/LGRS.2013.2252880. 
[35] G. Jian, "Reliability-Map-Guided Phase Unwrapping Method," IEEE Geoscience and Remote Sensing Letters, vol. 13, no. 5, pp. 716-720, May 2016. Doi: 10.1109/LGRS.2016.2539298.

[36] H. Wang, L. Tong, Y. Li and F. Xiao, "Phase Unwrapping Algorithm Based on Improved Weighted Quality Graph," IGARSS 2019 - 2019 IEEE International Geoscience and Remote Sensing Symposium, Yokohama, Japan, pp. 1769-1772, 2019. Doi: 10.1109/IGARSS.2019.8898671.

[37] M. Arevalillo-Herraez, F. R. Villatoro, and M. A. Gdeisat, "A Robust and Simple Measure for QualityGuided 2D Phase Unwrapping Algorithms," IEEE Transactions on Image Processing 25(6), 26012609, 2016. Doi: 10.1109/TIP.2016.2551370.

[38] M. Zhao et al., "Quality-guided phase unwrapping technique: comparison of quality maps and guiding strategies," Applied Optics 50(33), 6214, 2011. https://doi.org/10.1364/AO.50.006214.

[39] M. Costantini. "A novel phase unwrapping methodbased on network programing". IEEE Transactions on Geoscience and RemoteSensing, Vol 36, No 3, pp 813-821, May 1998. Doi: 10.1109/36.673674.

[40] Z. Yan and X. ZiJian, "A Hybrid Phase Unwrapping Algorithm Based on Quality-Guided and SurfaceFitting," 2018 IEEE International Workshop on Electromagnetics: Applications and Student Innovation Competition (iWEM), Aug. 2018. Doi: 10.1109/iWEM.2018.8536658.

[41] Z. Wang, A. C. Bovik, H. R. Sheikh, and E. P. Simoncelli, "Image Quality Assessment: From Error Visibility to Structural Similarity," IEEE Transactions on Image Processing, vol. 13, no. 4, pp. 600612, Apr. 2004. Doi: 10.1109/TIP.2003.819861. 